June 10, 2019. REVISED VERSION .

\title{
HDsEMG activity of the lumbar erector spinae in violin players:
}

\section{Comparison of two chairs.}

\section{Alessandro Russo, Alejandra Aranceta-Garza, Samuel D'Emanuele, Francesca Serafino, Roberto Merletti}

M2019-0259 -2-3-19 3851 words 5 figs 4 tables 1 app
IRB (YES line 66)
Informed consent- (yes line 68)
Length $-\mathrm{OK}$

Tables/figs - 10 total, reduce if possible Funding- see title page file

Conflict of interest- none

Prior presentation - none

\section{Abstract}

The purpose of this study was to compare an "ergonomic" alternative chair (A-chair), with a standard orchestra chair (O-chair) used by a group of nine violin players. The features of the highdensity surface EMG (HDsEMG) of the lumbar erector spinae muscles (ESM) were used for the comparison. The violinists played the same pieces of music for 2 hours without interruptions, on each chair, in two different days, one week apart. HDsEMG was recorded for 20s every 5 minutes using two electrode arrays of 16x8 electrodes each, one on each side of the spine and placed between the T11 and L4 levels. The sEMG was non-stationary and burst-like patterns were observed on 8 out of 9 violinists. The mean RMS and mean spectral frequency (MNF) value over the region of activity (ROA), the centroid of the ROA, the rates of change in time of the spatial mean of the RMS and MNF values, and the burst frequencies associated to the two chairs, were compared. Statistically significant reductions of RMS were observed in each violinist between the O-chair and A-chair (range between 11.80\% and 78.36\%). No significant changes of other spatial or spectral sEMG features were globally observed versus time or between chairs but were demonstrated by some subjects.

It is concluded that the A-chair is associated to a decrease of the sEMG amplitude of the ESM without changes of the spatial and temporal patterns of muscle activation.

\section{Introduction}

The sitting or standing posture assumed by performing musicians has considerable impact on their performance, breathing, muscle activity and back pain ${ }^{(1)}$ (2) (3). The activity of lumbar extensors muscles has been recently investigated by Ringheim et al. ${ }^{(4)}$ in subjects with and without low back pain, sitting for 30 min, using High Density sEMG (HDsEMG). 
Musicians playing string instruments are a small professional category with a high prevalence of playing-related musculoskeletal disorders (PRMD) ranging from $73.3 \%$ to $87.7 \%{ }^{(5)}$ mostly concerning upper extremities and back. In 1995, Cram et al. claimed that: "static working conditions, coupled with poor or inappropriate body mechanics, may cause prolonged tension in specific muscle groups. This, in turn, leads to fatigue, eventual muscle strain, and a myogenic ethiology of pain" (6). It is known that low muscle contraction levels sustained for long periods of time cause inflammation and pain ${ }^{(7,8)}$. This is the case of the erector spinae of sitting violinists. More recently, some authors ${ }^{(9)}$ claimed that chairs with appropriate back support may prevent the development of PRMD.

Quantitative assessments and comparisons of postures and chairs are lacking. Few previous studies investigated the erector spinae muscles of sitting workers and their pain mechanism using EMG electrode pairs ${ }^{(10,11)}$. More recently, other authors used electrode arrays or grids up to 128 contacts $(12-16)$

HDsEMG provides information about the spatial distribution of the sEMG and the region of activity (ROA) of a muscle. measured on the skin. In a previous preliminary study ${ }^{(17)}$, biomechanical (pelvic tilt, lumbar lordosis and thoracis kyphosis), and short term (5 min) HDsEMG measurements (spatial average of the EMG RMS value) were used to compare sitting of violinists and violists on a standard orchestra chair and on a series of different chairs (Varier Move and Varier $H A G$ with and without lumbar support). One of the Varier chairs appeared to be preferable to the others on the basis of sEMG and the biomechanical angles mentioned above. This chair (Varier Move with lumbar back rest adapted to each subject) was used in this study to further our understanding on the lumbar activity of violinists (A-Chair).

Three research questions are addressed in this work:

1. Is HDsEMG a suitable tool to detect and quantify sEMG differences in the lumbar erector spinae muscles due to two types of chairs used by violin players?

2. Are the two chairs associated to different values or time trends of sEMG features, detectable with the HDsEMG techniques, over long playing sessions?

3. Are myoelectric manifestations of fatigue detectable and measurable during such sessions?

To answer these questions, the objectives of this study were to compare the standard orchestra chair (O-chair) without back rest with an alternative chair (A-chair) presumably more "ergonomic" 
(M, Varier Move model with additional back rest, Varier Furniture Srl, http://www.varierfurniture.com), selected from a previous study ${ }^{(17)}$. This was done by acquiring HDsEMG data during a long period (2 h), to quantify long term sEMG amplitude and signal structure changes attributable to the two chairs. This study is the first using 128 electrodes on each side of the lumbar spinae of musicians, allowing for a larger recording area and limiting the truncation effect at the edges of the array ${ }^{(18)}$.

\section{Materials and methods}

\subsection{Subjects and protocol}

Nine right handed violinists ( 8 females, 1 male), participated in the study. None of them presented any history of chronic lower back pain or other back disorders. None of them was involved in the previous study ${ }^{(17)}$. All musicians provided informed consent prior to the tests. All the procedures used in this study were performed in accordance with the Helsinki Declaration of 1975, as revised in 2000 and 2008, and approved by the Italian National Health Service (ASL1 Torino 2002). Table 1 shows the demographic and anthropomorphic data of the nine subjects.

\section{Table 1 about here.}

The nine violinists played for two hours (with no interruptions) two standard pieces. This long time was expected to induce measurable myoelectric manifestations of muscle fatigue. The subjects did not perform other physically demanding activities in the same day before the test.

The two musical pieces selected were well known and deemed as demanding by the assessed group: 1. Kreutzer Study N 9 from 42 studies for violin as revised by Ivan Galmian (2min and 30s).

2. Kreutzer Study N 13 from 42 studies for violin as revised by Ivan Galmian (3min and 40s). The test was repeated in two different days, at least one week apart, using the O-chair on the first day and the A-chair on the second day. The A-chair had movable lumbar support which was adjusted to each musician according to their height (see Fig. 1).

Every 5 min, the musicians switched to a standard music piece (Rode, study N 2 of 24 Capricci for violin as revised by Ivan Galmian) which was played for $20 \mathrm{~s}$ during which sEMG recordings were acquired. This music piece was selected as it is a standard piece familiar to any violinist regardless of their expertise. This ensured that the musicians were always playing the same piece whilst HDsEMG was acquired. During each two-hour testing session, a total of 25 recordings of $20 \mathrm{~s}$ each 
(one every $5 \mathrm{~min}$ ) were used to monitor the time trends of sEMG features associated to each subject and each chair. Since no statistically significant trends of sEMG features were observed (see Results), the 25 EMG recordings were considered as repeated measurements per subject.

\subsection{Electrode placement, skin treatment, HDsEMG feature, recording and processing}

The skin was treated with abrasive paste (NuPrep, Skin Prep Gel), and cleaned with a wet cloth. Two electrode grids were placed, as indicated in Fig. 1 and Fig. 2, on each side of the spine at the lumbar level using T11 and L4 as anatomical landmarks ensuring consistency of position across participants and across trials. Each grid was composed of four smaller grids and had 16x8 electrodes (128 electrodes on each side of the spine) of $3 \mathrm{~mm}$ diameter (surface $=7 \mathrm{~mm}^{2}$ ) and spaced with inter-electrode distance (IED) of 10mm, as shown in Fig. 1d. Longitudinal differential signals were collected along the column direction (approximate fiber direction of the lumbar erector spinae) using the OT Bioelettronica 400 channel amplifier featuring $1 \mu \mathrm{V}_{\mathrm{RMS}}$ input referred noise, $\mathrm{CMRR}=95 \mathrm{~dB}$, bandwidth of 10-500 Hz, input impedance $>90 \mathrm{M} \Omega$ over the $10-500 \mathrm{~Hz}$ bandwidth, 16 bit A/D conversion, sampling frequency $=2048 \mathrm{~Hz}$, gain $=500$ and input resolution $=0.5 \mu \mathrm{V}$.

\section{Fig. 1 and 2 about here}

Each ROA, provided by each electrode grid for each of the 25 repetitions, was defined using the "active contours" method ${ }^{(19)}$ available in the Matlab 10 package. The active contours algorithm uses an initial user-defined contour that evolves and shrinks until a certain mathematical stop condition is met.

As observed in a previous study ${ }^{(17)}$, eight out of nine subjects presented intermittent burst-like activity of the ESM. The ninth-Subject 4 did not show any detectable amplitude modulation pattern. These bursts were investigated in this study with a novel identification and counting algorithm (see Appendix).

The sEMG signals of the individual channels were, in general, non-stationary because of the burst pattern (Fig. 3). The reported RMS values of the individual channels were estimated over epochs of $20 \mathrm{~s}$. The power spectral densities (PSD or power spectrum) and their mean frequencies (MNF or centroid frequency) were obtained as averages of spectra estimated over 40 1-s epochs (Welch method, $50 \%$ overlap) of each channel. In particular, the MNF values were unquestionably affected by noise and by the non-stationary nature of the signals (section 5.2). Estimates of spectral features, 
136 in this work, are averages strictly used for comparing the tested chairs and non-stationarities were 137 ignored.

The following features were computed from the sEMG signals over each of the twenty-five 20 -s epochs and used to compare spatial and temporal patterns associated to the two chairs:

1. Mean spatial value of the RMS maps of the SD signal over the ROA (this value will be referred to as RMS in the following). Mean spatial value of the MNF maps of the SD signal over the ROA (this value will be referred to as MNF in the following).

2. Centroid, or center of mass (CM), of the ROA. The effect of chair, side and time on the 145 coordinates $\mathrm{X}_{\mathrm{CM}}$ and $\mathrm{Y}_{\mathrm{CM}}$ was investigated by a 3-way ANOVA (Factors: chair type, side, time).

3. The slopes of the regression lines of RMS and of mean spectral frequency (MNF) versus time (25 measures over two hours) were considered as indicators of changes in time. They were normalized with respect to their initial values and expressed in \%/hour (see Results).

4. The burst frequency was estimated using the algorithm described in the Appendix.

The issue of amplitude normalization of sEMG is controversial, in particular for HDsEMG. In many previous works, when a single channel was recorded, the sEMG RMS value produced at the maximal voluntary contraction (MVC) was used as a normalization value (for example in Brandt et al. ${ }^{(20)}$, among many others). When an electrode grid is used, the issue is more complex and has not been investigated. The ROA and its centroid are very different at low contraction level with respect to the MVC level, reflecting the different structures involved in the two cases. This

\subsection{Interference and noise levels}

Power line and electrocardiographic (ECG) interference observed in the monopolar recordings of a previous study ${ }^{(17)}$ were not present in the differential recordings of this study. RMS maps with mean values below $6 \mu \mathrm{V}$ did not allow the definition of a ROA. Quantification of baseline signal values and their possible time trend (due to drifts of the electrodeskin interface) was important in order to classify the signals either as sEMG or as noise. For this purpose, a separate test was performed on a group of five subjects laying prone and relaxed on a bed for one hour. Surface signals were recorded with the same electrode setup and procedure as for the musicians. These estimates of the spatial mean RMS value of the noise maps provided a global 
average of $2.61 \mu \mathrm{V}_{\mathrm{RMS}}$ with a st. dev. of $0.46 \mu \mathrm{V}_{\mathrm{RMS}}$ (range: $1.90-3.30 \mu \mathrm{V}_{\mathrm{RMS}}$.). The background noise level was taken as $4 \mu \mathrm{V}_{\mathrm{RMS}}$ (obtained as the mean +3 st. dev.). Time trends were occasionally evident, and in some cases significantly different from zero, suggesting that comparable significant trends observed in some subjects were attributable to noise drifts.

The global average values of sEMG RMS ranged from $3.9 \mu \mathrm{V}_{\text {RMS }}$ to $18.8 \mu \mathrm{V}_{\text {RMS. Peak to peak sEMG }}$ values were in the range of $50-200 \mu \mathrm{V}$. The noise measurements confirmed an acceptable Signal/Noise ratio for the sEMG detected from the ESM during bursts. Fig. 3 and Fig. 4 show samples of raw signals (one column of the array) and demonstrate their good quality. Motor unit action potentials propagating in the vertical direction confirm their origin in the ESM.

\subsection{Measurement of subcutaneous adipose tissue}

Subcutaneous adipose tissue (SAT) thickness affects sEMG RMS values ${ }^{(21)}$. In our case this would hinder the comparison between right and left sEMG amplitudes. SAT thicknesses were measured by three operators, to check differences between three measurement sites (T11, L1, L3) and between left and right side, using an ultrasound scanner (Echo Blaster 128, Telemed, Lithuania). No significant differences were found using the ANOVA test with two factors: right and left side (R, L), anatomical levels of measurement of each subject $(\mathrm{N}=9$ measurements per side and per subject). Median thicknesses were $7.5 \mathrm{~mm}$ on the right and left sides. The lack of significant differences between side thicknesses indicates that RMS differences between sides (if any) are not be attributed to SAT.

\subsection{Burst frequency counts}

The bursts observed on the sEMG signals were counted using a novel algorithms using information from the entire electrode grid. The parameters of the algorithm were previously tested using 12 sEMG recordings, each of $20 \mathrm{~s}$ duration. The resulting 12 counts were compared with those provided by four human experts who did the 12 counts manually. See Appendix.

\subsection{Statistical analysis}

All the statistical analyses were carried out with Matlab and SPSS. The sEMG features respectively associated to the two chairs were compared using the Wilcoxon Signed Rank Test (Non-Gaussian 
203 data distribution) unless indicated otherwise. Paired t-tests were used after verification of normality 204 of the data distribution (Kolmogorov-Smirnov and Shapiro-Wilk test).

205 The spatial means of the RMS of the ROAs associated to sides (R, L) and chairs (O-chair, A-chair) 206 were computed for each $20 \mathrm{~s}$ test. For each of the nine violinists, the differences $\mathrm{RMS}_{\mathrm{R}}-\mathrm{RMS}_{\mathrm{L}}$ and $207 \mathrm{RMS}_{\mathrm{O}}-\mathrm{RMS}_{\mathrm{A}}$ were compared using the Wilcoxon paired Signed Rank Test. A similar analysis was 208 performed for the burst counts $\mathrm{B}_{\mathrm{R}}-\mathrm{B}_{\mathrm{L}}$ and $\mathrm{B}_{\mathrm{O}}-\mathrm{B}_{\mathrm{A}}$ using two-sided paired t-tests. A two-sided t209 test on the normalized slopes of the RMS regression lines was applied to detect significant 210 differences from zero (positive or negative trends). Normalized slope was defined as the slope of 211 the regression line of the feature of interest divided by the initial value (intercept with the Y-axis) 212 and was expressed as \%/s. The mean absolute displacement, along the $\mathrm{X}$ and $\mathrm{Y}$ coordinates, of the 213 ROA centroid was tested between chairs and sides for each subject along the two hours.

\section{Results}

\subsection{Raw signals quality and features}

217 Fig. 3 and 4 provide examples of signal quality. No effect of pressure against the back rest was 218 evident. The signals from most electrode pairs of the grid were not stationary and presented burst219 like activity as observed by ${ }^{(17)}$ on the same muscles. These burst-like patterns in the longitudinal single differential EMG signal were observed in 8 out of 9 violinists with bursts lasting 100-300 ms and repeating about 2.6-2.8 times per second. In Fig. 3, a 4-s recording selected out of a 20 s test, depicts raw sEMG from the same subject sitting on the O-chair and on the A-chair. Marked synchronization between the bursts of the right and left ESM is evident, as well as a reduction of the active motor unit pool on the A-chair, leading to a reduction of RMS values. Fig. 4 shows one burst-like pattern (zoom of Fig. 3) where propagating and non-propagating components of motor unit action potentials are evident and background activity (between bursts) is small. Burst behavior confirms previous observations on postural muscles (gastrocnemius) ${ }^{(22)}$ and deserves further investigation (see section 4.3). The nature and origin of the bursts are not discussed in this work and require more attention.

231 Contrary to expectations no significant correlation was observed between RMS values and SAT. 232 This may be due to the limited number of subjects. 
3.2 Changes of global sEMG features and myoelectric manifestations of muscle fatigue associated to the chairs.

Amplitude features. Fig. 5 shows an example of RMS maps and ROAs computed (over a $20 \mathrm{~s}$ epoch) on the right and left side of a violinist, for the O-chair and A-chair, at the beginning and at the end of two hours of playing. ROAs could be identified when the average RMS voltage over the grid was $>6 \mu \mathrm{V}_{\text {RMS. }}$. As indicated in Fig. 6 and Table 2, the mean RMS for the A-chair was lower than that for the $\mathrm{O}$-chair in each of the nine violinists. The mean percent decrement ranged from $16.59 \%$ to $72.49 \%$ with an average of $40.38 \%$ (Wilcoxon Signed Rank test, $\mathrm{p}<0.05$ for each subject, $N=25$ measurements over two hours). Some subjects presented significant positive or negative trends (Table 3). The regression slopes of the RMS values over time were in the range of $3 \mu \mathrm{V}_{\mathrm{RMS}} / \mathrm{h}$ to $+1.2 \mu \mathrm{V}_{\mathrm{RMS}} / \mathrm{h}$. These slopes are comparable with the RMS regression slopes due to noise drifts observed in the five relaxed subjects lying prone on a bed $(-0.36 \mu \mathrm{V} / \mathrm{h}$ to $+0.76 \mu \mathrm{V} / \mathrm{h})$. Globally, the averaged (across subjects) RMS slopes of the relaxed subjects and of the violinists were not significantly different from zero and from each other.

Fig. 5 and 6 about here. Table 2 about here.

Spectral features. The regression slopes of MNF values over time were in the range of $-34.8 \mathrm{~Hz} / \mathrm{h}$ to $+12.6 \mathrm{~Hz} / \mathrm{h}$ for the relaxed subjects and in the range of $-6.6 \mathrm{~Hz} / \mathrm{h}$ to $+28.8 \mathrm{~Hz} / \mathrm{h}$ for the violinists.

The averaged MNF slopes of the relaxed subjects and of the violinists were not significantly different from zero and from each other. As shown in Table 3, some subjects showed positive trends and some showed negative trends in the values of RMS or MNF, however, no consistent behavior could be observed across subjects (see section 4.2).

sEMG non-stationarity. Both RMS and MNF values were affected by the non-stationary burst-like sEMG patterns. These patterns were not detected in the relaxed subjects and are likely associated to playing the violin; however, they were not affected by the rhythm and speed of the music, by time or by the chair used. Despite the estimates of average amplitude and spectral features of nonstationary signals, comparison of RMS and MNF values between chairs, in identical conditions, was considered acceptable (see section 4.2).

The values of MNF and burst frequency revealed different individual responses (with some cases of statistically significant difference) between chairs, as reported in Table 4 . The differences between burst frequencies associated to the two chairs were found to be small (less than $6 \%$ between means), 
and the global mean response did not seem to adequately represent the responses of individuals. The same considerations apply to the results reported in Table 3 concerning the slopes of RMS and MNF. The physiological significance of these different individual behaviors should be further investigated.

Centroid of the ROA. ANOVA multivariate analysis was applied to a) identify significant changes in the location of the CM of the ROA versus time and, b) to test if the coordinates of the CM were significantly affected by side or chairs. Paired t-tests were performed on $\mathrm{X}_{\mathrm{CM}}$ and $\mathrm{Y}_{\mathrm{CM}}$ coordinates after images were interpolated by a factor of 15 , and after verifying normality of the $\mathrm{X}_{\mathrm{CM}}$ and $\mathrm{Y}_{\mathrm{CM}}$ distribution (Shapiro-Wilk test). No significant change of the location of the centroid of the maps could be observed, either versus time, side, or chair type.

\section{Table 3 and 4 about here}

\section{Discussion}

\subsection{Quality of signals and of their features}

It is well known that comparisons of the amplitude features of sEMG between muscles, subjects, or tasks are highly critical ${ }^{(23)}$. Spectral features are even more critical than amplitude features. As a consequence, considerations of individual behaviors (Fig. 6) should be preferred to considerations based on averages (Table 2). In this work, we performed paired comparisons of sEMG features (within subject, for one muscle and one task) associated to two different chairs being tested in two different days at least one week apart. It was not possible to blind musicians from the types of chairs. used; nonetheless, it was unlikely to introduce bias given the objective endpoints (failure of task or fatigue). In addition However, the two tests were performed at least seven days apart to avoid effects of one on the other. Of importance, Schinkel-Ivy et al (24) demonstrated that the erector-spinae muscles (ESM) display similar trends and repeatable sEMG measures in test-retest trials.

\subsection{Changes of sEMG features attributable to the chairs}

A statistically significant decrease of the sEMG amplitude (RMS) of the ESM was the main difference observed when subjects were sitting on the A-chair. when compared to the A-chair. The average reduction with respect to the O-chair was about 40\%. Fig. 1 shows that the trunk-thigh 
angle was greater when sitting on the A-chair with respect to sitting on the O-chair; this is likely one of the reasons for the observed amplitude changes. The same chair was used in a previous study by Cattarello et al. ${ }^{(17)}$ with the same trunk-thigh angle (but without back support). A reduction of about $20 \%$ of RMS was reported suggesting a role of the back-rest in determining sEMG amplitude of ESM.

The observed reduction of RMS values from the $O$-chair to the $A$-chair is due to a change of sEMG amplitude over the ROA without This finding was associated to small non-significant changes of the shape or size or location of the ROA or of the burst patterns. It might indicate a change in the load sharing among the muscles of the lumbar back with a possible reduced role of the ESM and a greater role of deeper muscles, such as the multifidus, whose contribution to the sEMG is small. Of interest, Ringheim et al ${ }^{(14)}$ observed periodic oscillations of activity between the right and left ESM at a frequency around 8 per minute. These oscillations were observed by Ringheim et al. during sustained sitting but were not observed in our study.

The lack of myoelectric manifestations of muscle fatigue is puzzling (the musicians perceived tiredness after $2 \mathrm{~h}$ of playing) and may be due to their training level. In addition, the contraction level of the ESM was deemed low and below the "fatigue threshold" discussed by McCrary ${ }^{(25)}$ and defined as "the power, torque, or force at which the rate of change of sEMG amplitude is zero and below which neuromuscular fatigue is negligible and unpredictable".

Finally, the contraction of the ESM of a sitting musician involves only a limited number of fatigue resistant motor units, likely within the pool of the so-called "Cinderella motor units" as proposed by Hägg ${ }^{(7,8)}$. The behaviour of these motor units must be investigated by sEMG decomposition ${ }^{(26)}$ in order to identify whether the motor unit pool is stable or if motor unit substitution/rotation is present.

The "fatigue" perceived by the musicians at the end of the performance has an origin likely not associated to the electrophysiology of the muscles and deserves further investigation ${ }^{(27) .}$

\subsection{Burst analysis}

The finding of burst-like modulation of sEMG amplitude (Fig. 3 and 4, Table 4) confirms previous observations ${ }^{(17,22)}$. The small positive or negative differences between burst frequencies associated to the two chairs and among subjects, suggest that such pattern derives from the postural control system rather than from the adopted chair. Such intermittent control mechanism is likely a background physiological strategy and must be investigated further. 


\section{5. Conclusions and limitations of the study.}

\subsection{Conclusions}

Three major observations and conclusions derive from this investigation:

1. In nine out of nine sitting violinists the sEMG RMS value of the ESM were significantly lower when the musician was sitting on a saddle chair (A-chair, with lumbar back rest and a hip angle of $105^{\circ}-135^{\circ}$, see Fig. 1) with respect to sitting on a standard orchestra chair (O-chair, no back rest). The average decrease found was $40.1 \%$.

2. No global significant/consistent trends of RMS or MNF were detected on the nine violinists while playing for $2 \mathrm{~h}$. Individual significant trends were manifested by some subjects but most may be attributed to baseline drifts as they were observed in resting subjects as well. The perception of fatigue does not seem to have an electrophysiological counterpart. This is likely due to the low contraction level and to the exposure that the musicians have to many weekly hours of practice for many years ${ }^{(14)}$.

3. The sEMG of the ESM showed a burst-like amplitude modulation in 8 out of 9 violinists (with an average rate of about 2.60 bursts/s) confirming previous observations ${ }^{(17)}$. The burst mechanism deserves further investigation. The contraction level of the ESM was likely below the "fatigue threshold" discussed by McCrary ${ }^{(25)}$.

Normalization of sEMG. Because of limited time availability and lack of literature reports concerning normalization of 2D sEMG signals, no normalization procedure was applied. Recommendations for proper normalization modalities are lacking and should be developed for 2D sEMG signals. Ambient conditions, such as room temperature and humidity, were not measured but were maintained to comfortable values by the air conditioning system. 
Measurements were not randomized. For organizational reasons the O-chair was tested first and the A-chair was tested a week later. It is unlikely that there would be any influence of the first measurement over the second.

The sEMG RMS values, estimated every 5 min over $20 \mathrm{~s}$ long epochs and averaged over the ROA, ranged from $4 \mu \mathrm{V}$ to $19 \mu \mathrm{V}$ (Fig. 6). Because of these low sEMG amplitude levels, it was necessary to estimate the noise baseline. This is usually done by measuring sEMG RMS in relaxed conditions before and/or after a test. The limited availability of time by the violinists did not allow this procedure. Noise was therefore estimated from the same muscles, using the same electrode setup, from five healthy subjects in the same age range lying prone on a bed for $1 \mathrm{~h}$. This test indicated that RMS noise baseline was $2.6 \mu \mathrm{V}_{\mathrm{RMS}}$ with a st. dev. of $1.4 \mu \mathrm{V}_{\text {RMS. }}$ The most caudal channels (e.g. the bottom channels in Fig. 3b) had RMS of about $4 \mu \mathrm{V}$ corresponding to the mean +1 st. dev. of the 65 measurements taken on the five relaxed subjects (13 measurements per each of the 5 subjects). The value of $4 \mu V_{\text {RMS }}$ was therefore taken as baseline noise.

Another limitation has to do with the sampled population, as it was not homogeneous and deemed limited to allow associations of sEMG behaviours to age, gender, experience and training schools. Therefore, inter-subject variations were not investigated in this study.

Violinists were studied only in the sitting position on two different chairs. The subjects played at the speed of their choice, without a metronome. The possible association between: sEMG amplitude, spectral variables, and burst rate on one hand, and the type of music played, on the other hand, were not investigated because the work was mainly focused on the comparison of the sEMG features of the ESM associated to two types of chairs. The physiological mechanisms possibly explaining our findings and observations (i.e. burst-like activity) have not been addressed. Standard spectral analysis, adopted in this work, is usually applied to stationary signals but does not "require" stationarity if average values of RMS and MNF are acceptable. Approaches more suitable for non-stationary signals (such as time-frequency representations) would track the bursts but just shift the problem of defining one average value for RMS and MNF over each of the $20 \mathrm{~s}$ observation intervals. Although the spectral analysis is not rigorous because of the non-stationary signals, it allows comparison between the two chairs under test. 


\section{References}

1. Ackermann BJ, O'Dwyer N, Halaki M. The difference between standing and sitting in 3 different seat inclinations on abdominal muscle activity and chest and abdominal expansion in woodwind and brass musicians. Front Psychol. 2014;5:913. doi: 10.3389/fpsyg.2014.00913. eCollection 2014.

2. Price K, Schartz P, Watson AH. The effect of standing and sitting postures on breathing in brass players. Springerplus. 2014 Apr 28;3:210. doi: 10.1186/2193-1801-3-210. eCollection 2014.

3. Baadjou VA, van Eijsden-Besseling M, Verbunt J, de Bie RA, Geers R, Smeets R, Seelen H. Playing the Clarinet: Influence of Body Posture on Muscle Activity and Sound Quality. Med Probl Perform Art. 2017;32(3):125-131. doi: 10.21091/mppa.2017.3021.

4. Ringheim IA-Ohoo, Indahl A, Roeleveld K. Reduced muscle activity variability in lumbar extensor muscles during sustained sitting in individuals with chronic low back pain. PLoS ONE 14(3): e0213778. https://doi.org/10.1371/journal.pone.0213778

5. Zaza C. Playing-related musculoskeletal disorders in musicians: a systematic review of incidence and prevalence. CMAJ: Canadian Medical Association Journal. 1998;158(8):1019-25.

6. Cram JR, Vinitzky I. Effects of chair design on back muscle fatigue. Journal of occupational rehabilitation. 1995;5(2):101-13.

7. Hagg G. Static work load and occupational myalgia-A new explanation model. Anderson, D Hobart and J Danoff (ed) Electromyographical Kinesiology Elsevier Science Publishers, Amsterdam: 141-144. 1991.

8. Hagg GM. Human muscle fibre abnormalities related to occupational load. Eur J Appl Physiol. 2000;83(2-3):159-65.

9. Foxman I, Burgel BJ. Musician health and safety: Preventing playing-related musculoskeletal disorders. Journal of the American Association of Occupational Health Nurses. 2006;54(7):309-16.

10. Mork PJ, Westgaard RH. Back posture and low back muscle activity in female computer workers: a field study. Clinical biomechanics (Bristol, Avon). 2009;24(2):169-75.

11. van Dieen JH, de Looze MP, Hermans V. Effects of dynamic office chairs on trunk kinematics, trunk extensor EMG and spinal shrinkage. Ergonomics. 2001;44(7):739-50.

12. Abboud J, Nougarou F, Loranger M, Descarreaux M. Test-Retest Reliability of Trunk Motor Variability Measured By Large-Array Surface Electromyography. Journal of manipulative and physiological therapeutics. 2015;38(6):359-64.

13. Farina D, Gazzoni M, Merletti R. Assessment of low back muscle fatigue by surface EMG signal analysis: methodological aspects. Journal of Electromyography and Kinesiology. 2003;13(4):319-32.

14. Ringheim I, Indahl A, Roeleveld K. Alternating activation is related to fatigue in lumbar muscles during sustained sitting. Journal of Electromyography and Kinesiology. 2014;24(3):380-6.

15. Falla D, Gizzi L, Tschapek M, Erlenwein J, Petzke F. Reduced task-induced variations in the distribution of activity across back muscle regions in individuals with low back pain. Pain. 2014;155(5):944-53.

16. Merletti R, Afsharipour B, Dideriksen J, Farina D. Muscle Force and Myoelectric Manifestations of Muscle Fatigue in Voluntary and Electrically Elicited Contractions. Surface Electromyography : Physiology, Engineering, and Applications: John Wiley \& Sons, Inc.; 2016. p. 273-310.

17. Cattarello P, Vinelli S, D'Emanuele S, Gazzoni M, Merletti R. Comparison of chairs based on HDsEMG of back muscles, biomechanical and comfort indices, for violin and viola players: A shortterm study. Journal of Electromyography and Kinesiology. 2018;42:92-103.

18. Afsharipour B, Soedirdjo S, Merletti, R. Two dimensional surface EMG: the effects of electrode size, interelectrode distance and image truncation. Biomedical Signal Processing and Control. 2019;49:298307. 
19. Caselles V, Kimmel R, Sapiro G. Geodesic Active Contours. International Journal of Computer Vision. 1997;22:61-79.

20. Brandt M, Andersen LL, Samani A, Jakobsen MD, Madeleine P. Inter-day reliability of surface electromyography recordings of the lumbar part of erector spinae longissimus and trapezius descendens during box lifting. BMC Musculoskelet Disord. 2017;18(1):519. doi: 10.1186/s12891-017-1872-y.

21. Kuiken TA, Lowery MM, Stoykov NS. The effect of subcutaneous fat on myoelectric signal amplitude and cross-talk. Prosthet Orthot Int. 2003;27(1):48-54.

22. Vieira TM, Loram ID, Muceli S, Merletti R, Farina D. Recruitment of motor units in the medial gastrocnemius muscle during human quiet standing: is recruitment intermittent? What triggers recruitment? Journal of neurophysiology. 2012;107(2):666-76.

23. Vigotsky AD, Halperin I, Lehman GJ, Trajano GS, Vieira TM. Interpreting Signal Amplitudes in Surface Electromyography Studies in Sport and Rehabilitation Sciences. Frontiers in physiology. 2017;8:985.

24. Schinkel-Ivy A, DiMonte S, Drake JDM. Repeatability of kinematic and electromyographical measures during standing and trunk motion: How many trials are sufficient? Journal of Electromyography and Kinesiology. 2015;25(2):232-8.

25. McCrary JM, Ackermann BJ, Halaki M. EMG amplitude, fatigue threshold, and time to task failure: A meta-analysis. Journal of science and medicine in sport. 2018;21(7):736-41.

26. Holobar A, Zazula D. Correlation-based decomposition of surface electromyograms at low contraction forces. Med Biol Eng Comput. 2004;42(4):487-95.

27. Weir JP, Beck TW, Cramer JT, Housh TJ. Is fatigue all in your head? A critical review of the central governor model. Br J Sports Med. 2006;40(7):573-586; discussion 586.

28. Bonato P, D'Alessio T, Knaflitz M. A statistical method for the measurement of muscle activation intervals from surface myoelectric signal during gait. IEEE transactions on bio-medical engineering. 1998;45(3):287-99.

29. Merlo A, Farina D, Merletti R. A fast and reliable technique for muscle activity detection from surface EMG signals. Ieee Transactions on Biomedical Engineering. 2003;50(3):316-23.

30. Gray H, Vandyke Carter H. Anatomy of the human body. Febiger L, editor. Philadephia, 1918.

\section{Acknowledgment}

\section{Blinded}


481 Table 1: Demographic and anthropometric data of the nine violinists and their musical career (years 482 playing the instrument), hours of playing per week and their subcutaneous adipose tissue (SAT)

483 thickness at the ESM level. Body Mass Index (BMI) is defined as: $B M I=m / h^{2}$ where $m$ is the 484 subject mass $(\mathrm{kg})$ and $h$ is the height $(\mathrm{cm})$. All subjects had right dominance. Subject 6 is a violin 485 teacher, all the other subjects were students.

486

\begin{tabular}{|c|c|c|c|c|c|c|c|c|}
\hline \multicolumn{9}{|c|}{ Violinists (N=9) } \\
\hline Subject & Gender & $\begin{array}{c}\text { Age } \\
(\text { years })\end{array}$ & $\begin{array}{c}\text { Weight } \\
(\mathrm{kg})\end{array}$ & $\begin{array}{c}\text { Height } \\
(\mathrm{cm})\end{array}$ & $\begin{array}{c}\text { BMI } \\
\left(\mathrm{kg} / \mathrm{m}^{2}\right)\end{array}$ & $\begin{array}{c}\text { Musical } \\
\text { career } \\
\text { (years })\end{array}$ & $\begin{array}{c}\text { Weekly } \\
\text { practice } \\
\text { (hours/ week) }\end{array}$ & $\begin{array}{c}\text { SAT } \\
\text { thickness } \\
(\mathrm{mm})\end{array}$ \\
\hline 1 & F & 22 & 50 & 156 & 20.55 & 10 & 6 & 7.60 \\
\hline 2 & F & 20 & 51 & 167 & 18.29 & 14 & 6 & 5.70 \\
\hline 3 & F & 18 & 55 & 165 & 20.20 & 9 & 9 & 9.30 \\
\hline 4 & F & 17 & 47 & 160 & 18.36 & 9 & 7 & 5.30 \\
\hline 5 & M & 16 & 60 & 172 & 20.28 & 11 & 10 & 5.60 \\
\hline 6 & F & $50 *$ & 62 & 163 & 23.34 & $40 *$ & $42 *$ & 10.40 \\
\hline 7 & F & 15 & 53 & 161 & 20.45 & 7 & 7 & 6.40 \\
\hline 8 & F & 22 & 50 & 165 & 18.37 & 14 & 12 & 7.60 \\
\hline 9 & F & 22 & 65 & 158 & 26.04 & 12 & 6 & 11.20 \\
\hline Mean & $\mathbf{8 F , ~ 1 M ~}$ & $\mathbf{1 9 . 0 0}$ & $\mathbf{5 4 . 7 7}$ & $\mathbf{1 6 3}$ & $\mathbf{2 0 . 6 5}$ & $\mathbf{1 1 . 0 0}$ & $\mathbf{7 . 8 7}$ & $\mathbf{7 . 6 0}$ \\
\hline
\end{tabular}

*indicates an outlier value not included in the calculation of (mean, st. dev.) of age, musical career and weekly practice. 
491 Table 2. Mean percentage decrement between O-chair and A-chair (with respect to the O-chair) of 492 the RMS spatial mean of sEMG computed over the ROA. Decrements are positive.

493 For each subject the mean and st.dev. of $100 \cdot\left(\mathrm{RMS}_{\mathrm{Oi}}-\mathrm{RMS}_{\mathrm{Ai}}\right) / \mathrm{RMS}_{\mathrm{Oi}}$ is computed for $1 \leq \mathrm{i} \leq 25$ 494 where $\mathrm{i}$ is the index of the measurements performed every $5 \mathrm{~min}$, over a $20 \mathrm{~s}$ epoch, for two hours. 495 The decrement of each subject is significantly different from zero (Wilcoxon Signed Rank test, $496 \mathrm{p}<0.05)$. See also Fig. 6.

497

\begin{tabular}{cccc}
\hline Subject & $\begin{array}{c}\text { Mean RMS percent } \\
\text { decrement on left side } \\
(\text { mean } \pm \text { st.dev) } \\
\mathrm{N}=25\end{array}$ & $\begin{array}{c}\text { Mean RMS percent } \\
\text { decrement on right side } \\
\text { (mean } \pm \text { st.dev) } \\
\mathrm{N}=25\end{array}$ & $\begin{array}{c}\text { Mean RMS percent } \\
\text { decrement, sides } \\
\text { merged } \\
\text { (mean } \pm \text { st.dev) } \\
\mathrm{N}=50\end{array}$ \\
\hline 1 & $28.27 \pm 5.96$ & $21.51 \pm 4.81$ & $24.89 \pm 5.41$ \\
\hline 2 & $78.36 \pm 3.03$ & $66.62 \pm 5.62$ & $72.49 \pm 4.51$ \\
\hline 3 & $62.93 \pm 4.04$ & $69.48 \pm 1.68$ & $66.20 \pm 3.09$ \\
\hline 4 & $47.83 \pm 14.02$ & $38.00 \pm 10.38$ & $42.91 \pm 12.33$ \\
\hline 5 & $61.06 \pm 4.44$ & $56.89 \pm 5.90$ & $58.97 \pm 5.22$ \\
\hline 6 & $27.45 \pm 12.98$ & $15.09 \pm 17.45$ & $21.27 \pm 15.37$ \\
\hline 7 & $11.97 \pm 7.11$ & $21.22 \pm 16.71$ & $16.59 \pm 12.84$ \\
\hline 8 & $19.14 \pm 8.02$ & $59.43 \pm 3.24$ & $39.28 \pm 6.11$ \\
\hline 9 & $22.77 \pm 7.41$ & $19.01 \pm 9.89$ & $20.89 \pm 8.73$ \\
\hline Total & $\mathbf{3 9 . 9 7 \pm 8 . 2 7}$ & $\mathbf{4 0 . 8 0} \pm \mathbf{9 . 9 5}$ & $\mathbf{4 0 . 3 8 \pm \mathbf { 8 . 7 2 }}$ \\
\hline
\end{tabular}

498

499

500

501

502

503

504

505 
507 Table 3. Number of statistically significant increases or decreases of individual RMS (RMS 508 slope count) and MNF (MNF slope count) versus time. Right and left side grids of the ESM 509 values are merged. NS: non-significant changes.

\begin{tabular}{|c|c|c|c|}
\hline & & $\begin{array}{r}9 \text { sul } \\
18 \text { regressions } \\
\text { (9 Right }\end{array}$ & $\begin{array}{l}\text { ects } \\
\text { oer chair type } \\
9 \text { Left) }\end{array}$ \\
\hline & & RMS slope count & MNF slope count \\
\hline \multirow{3}{*}{ 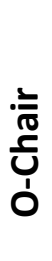 } & Significantly positive $\uparrow$ & 3 & 5 \\
\hline & Significantly negative $\downarrow$ & 8 & 0 \\
\hline & NS & 7 & 13 \\
\hline \multirow{3}{*}{ 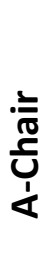 } & Significantly positive $\uparrow$ & 4 & 9 \\
\hline & Significantly negative $\downarrow$ & 8 & 1 \\
\hline & NS & 6 & 8 \\
\hline
\end{tabular}


Table 4. Violin players showing bursts. Comparison of mean burst frequency between the two chairs (A-chair; O-chair) and by side (L-Left; R-Right) of the erector spinae muscle.

* indicates statistically significant differences (two-sided paired t-tests $\mathrm{p}<0.05$ ),

$\mathrm{NS}=$ non-significant difference. Subject 4 does not show bursts.

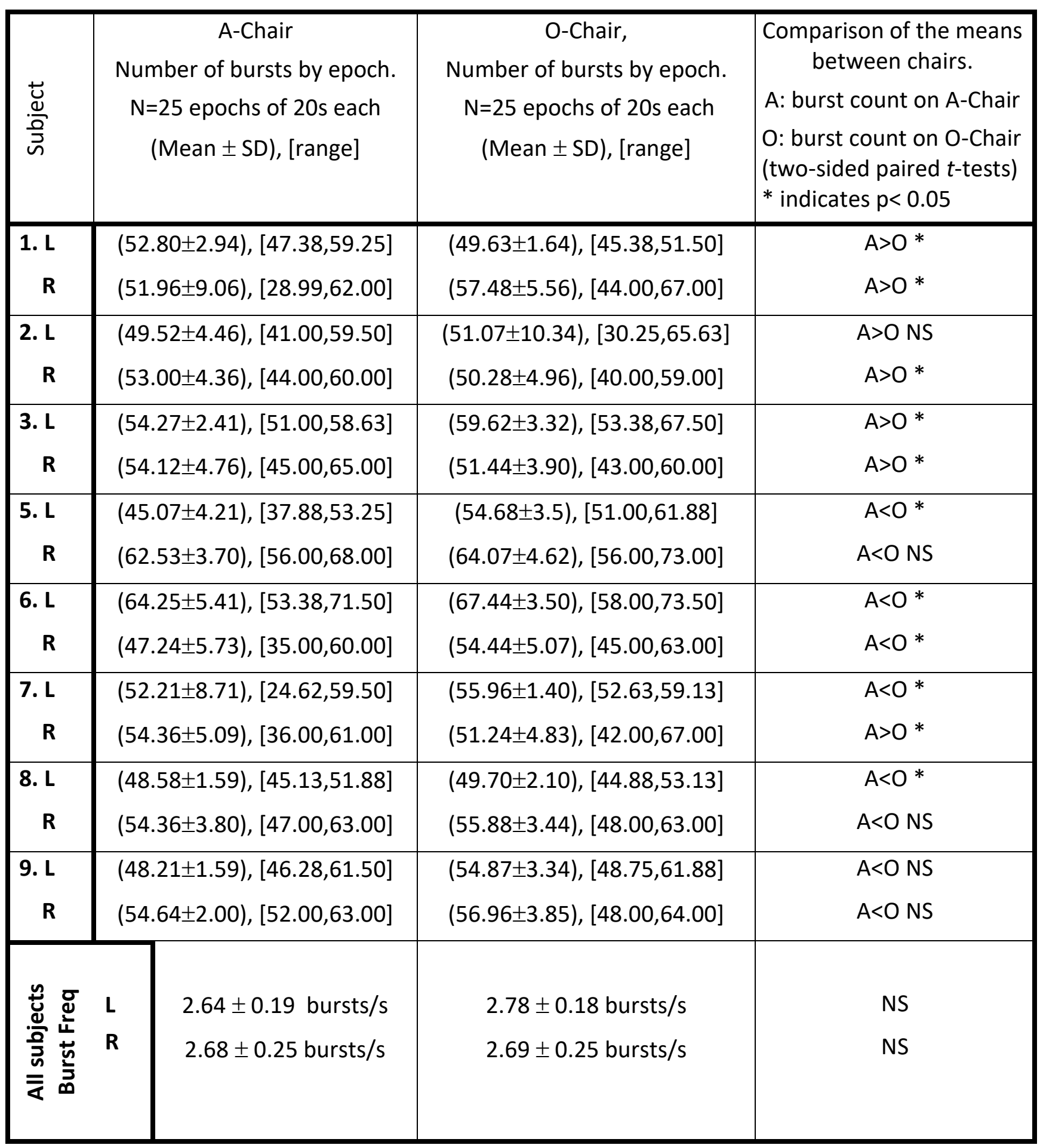




\section{Appendix: Burst detection algorithm.}

Algorithm. Many burst detection algorithms applicable to individual sEMG channels have been previously described in the literature ${ }^{(28,29)}$ but applications to multichannel array detection and systematic comparison with human counts is lacking. An algorithm based on HDsEMG detected with a 16x8 electrode grid placed on the ESM was developed for automatic counting of the bursts. The algorithm has been tested on 12 recordings selected from nine subjects according to the following criterion: four recordings showed clear bursts of sEMG activity, four showed less clear bursts, and four showed bursts that were just visually detectable (see below). Each multichannel recording lasted $20 \mathrm{~s}$ and consisted of $15 \times 8=120$ single differential channels. The algorithm is based on the following three steps:

Step 1. A moving average window of 60 samples (30ms), with 30 samples (15ms) overlapping was applied to each 20-s long longitudinal single differential squared signal (channel $r, c$ ) resulting in its power envelope $\left(R M S_{r, c}{ }^{2}(t)\right)$ sampled at 66.6 samples/s. The 15 signals $\left(R M S_{r, c}{ }^{2}(t)\right)$ of each column $c$ were averaged in space across the 15 rows to obtain $R M S_{c}^{2}(t)=\frac{1}{15} \sum_{r=1}^{15} R M S_{r, c}^{2}(t)$ for $1 \leq c \leq 8$, resulting in eight envelope signals per grid.

Step 2. A threshold $T$ was set at the median $\left(50^{\text {th }}\right.$ percentile) of the amplitude distribution of each envelope signal $R M S_{c}^{2}(t)$. The amplitude distribution consisted of $66.6 \mathrm{samples} / \mathrm{s} \times 20 \mathrm{~s}=1333$ samples. A binary signal $B 1_{c}(t)$ was created for each column $c\left(B 1_{c}(t)=0\right.$ or 1 for samples of $R M S_{c}^{2}(t)$ below or above T, respectively). Gaps in $B 1_{c}(t)$ shorter than $65 \mathrm{~ms}$ (4 samples) were forced to 1 . Bursts of 1 sample were forced to 0 . These values were selected empirically, by trial and error. The resulting binary signal $B 2_{c}(t)$ was used to count the bursts identified in each column.

Step 3. Since the eight burst counts on the eight columns of each array were never significantly different from each other (two-sided t-tests, $N=8, p>0.05$ ), the counts were averaged to obtain the burst count for each array and for each 20 -s recording.

Validation of the algorithm. Four 20-s recordings showing clear bursts (visual analysis), four 20-s recordings showing inter-bursts activity or noise, and four 20s recordings showing questionable bursts, were randomly selected among the recordings obtained from the eight subjects indicated in Table 4. 
556 Each of the 12 recordings was analysed by four experts who counted the bursts manually. The four 557 "human counts" (HC) were then compared with the counts provided by the algorithm (CC) (two558 way analysis of variance, ANOVA).

559

560 The maximal discrepancy among the four HCs was 5 bursts out of $46-59$ bursts $(<10.6 \%)$.

561 The difference between the average of the four HC and the single CC did not exceed \pm 1.75 burst 562 out of 46 -59 bursts (about $\pm 3.8 \%$ ) for any of the 12 recordings and was not statistically significant 563 (Paired samples t-test). It is concluded that the algorithm provided computer counts consistent with 564 the human count across the three groups of four signals of different quality.

565

566 
567

568

569

570

571

572

573

574

575

576

577

578

579

580

581

582

583

584

585

586

587

588

589

590

591

592

593

594

595

596

597

598

599

600

\section{Figure captions}

Figure 1: a) The violinist plays on the O-chair (standard orchestra chair) with the trunk erect, with feet at the same distance from the body with the extremities slightly diverging. Trunk-thigh angle is about $90^{\circ}$ and there is no torsion of the trunk.

b) The violinist plays on the A-chair with the trunk erect and trunk-thigh angle between $105^{\circ}$ and $135^{\circ}$. The back is always in contact with the lumbar support.

c) Example of electrode grid positioning on the lumbar portion of the right and left erector spinae muscles between spinal processes T11 and L4. Column numbering is reported under the first and last columns.

d) The grids have interelectrode distance IED $=10 \mathrm{~mm}$ and electrode diameter $\varnothing=3 \mathrm{~mm}$.

Figure 2: Anatomy of the back muscles at the lumbar level. The terminal portion of trapezius inferior, the tendinous part of latissimus dorsi and dentatus, overlap the lumbar portion of erector spinae. Image source: Gray's Anatomy book, $20^{\text {th }}$ Edition ${ }^{(30)}$.

Figure 3: Single differential signals from a violinist erector spinae muscle (ESM) whilst using the O-chair (a) and the A-Chair (b). The signals are detected from column 7 of the left ESM (top graph) and column 2 of right ESM (bottom graph) on a time window of $4 \mathrm{~s}$. These signals were recorded after one hour of playing. The RMS values calculated over the entire length of the signal (20 s) are reported on the left of each trace. Twelve bursts are clearly visible with duration of 200$250 \mathrm{~ms}$. The zoom of a burst is reported in Fig. 4.

Figure 4: Zoom of sEMG burst-like patterns on the single differential signal (as shown in Fig. 3) where a) corresponds to the O-chair and b) corresponds to the A-chair. Both graphs correspond to column 7 of left side of the ESM on a $250 \mathrm{~ms}$ time window. RMS values calculated over the entire length of the signal (20 s) are reported on the left side of each trace. Propagating motor unit action potentials (MUAPs, dotted lines) suggest that the signals originate from the erector spinae. Nonpropagating MUAPs suggest that the signals originate from end-of-fiber effects of the erector spinae or of other muscles.

Figure 5: Single differential RMS maps relative to subject 8 for the $O$ and A chair, at the beginning (5 minutes) and the end (120 minutes) of the test. Maps are computed on the entire length of the signals (20 s). The dark contour indicates the edge of the region of activity (ROA) identified by 
601 means of map segmentation ${ }^{(19)}$. The mean, minimum and maximum values of the ROA are

602 reported ( $\mu \mathrm{V}_{\mathrm{RMS}}$ in time) above each map. The centroid of each ROA, the colour scale (0-30

$\left.603 \mu \mathrm{V}_{\mathrm{RMS}}\right)$ and a schematic representation of the vertebrae $\left(\mathrm{T}_{11}-\mathrm{L}_{4}\right)$ are reported.

604

605 Figure 6: Mean RMS (computed over the ROA, left and right side merged together) for each 606 subject and each chair. a) at the beginning and at the end of the test, for O-chair. b) Same for A607 chair, c) global mean over the O-chair vs the global mean for the A-chair for each subject.

608 The subject number is reported next to each line. The noise level ( $\left.4 \mu \mathrm{V}_{\mathrm{RMS}}\right)$ and the mean value 609 below which no segmentation was possible (about $6 \mu \mathrm{V}_{\mathrm{RMS}}$ ) are indicated. The noise level is 610 defined as the spatial mean + 3 st. dev of the EMG RMS values detected from five subjects lying 611 prone on a bed for one hour.

612 

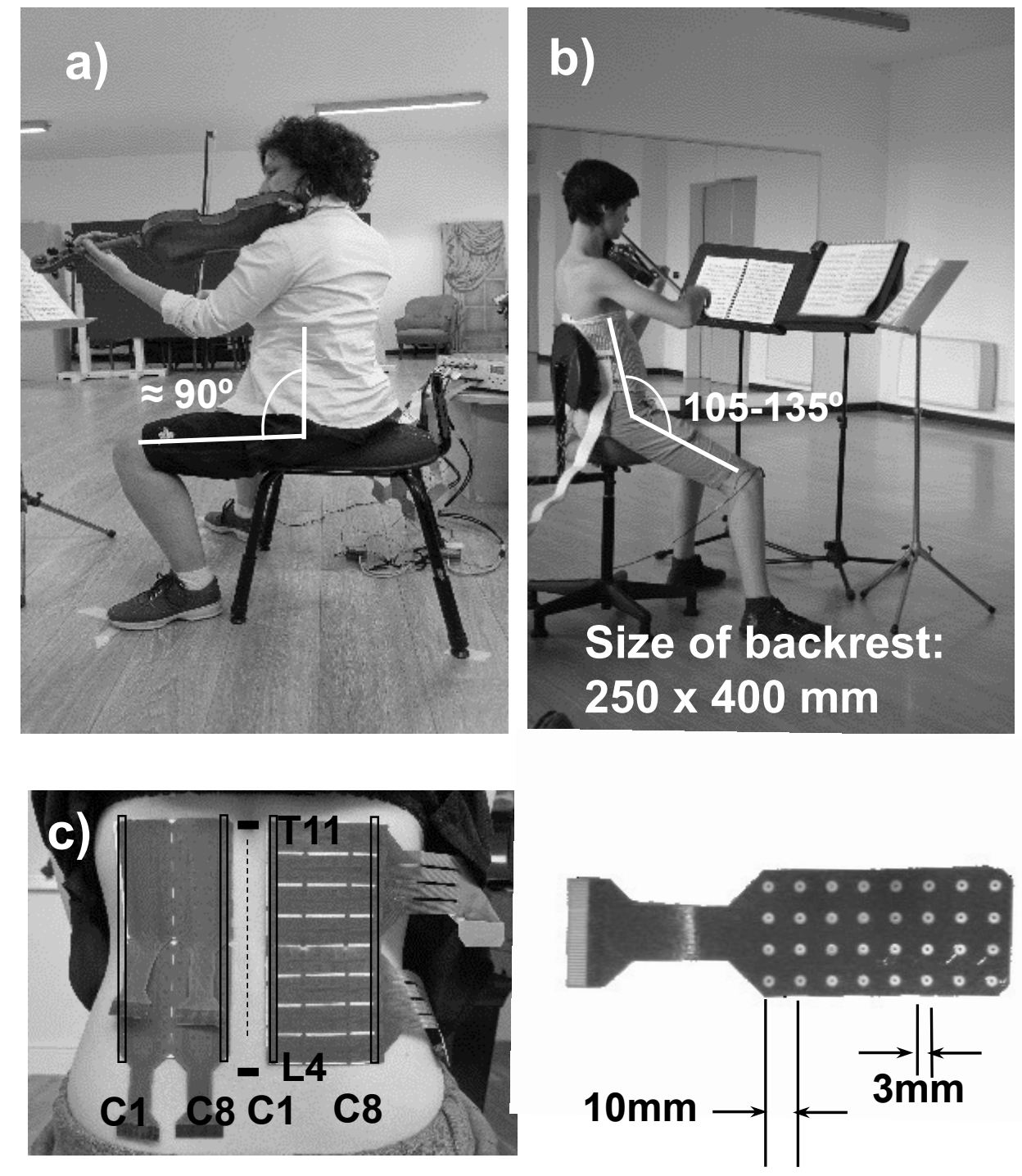

Fig 1 


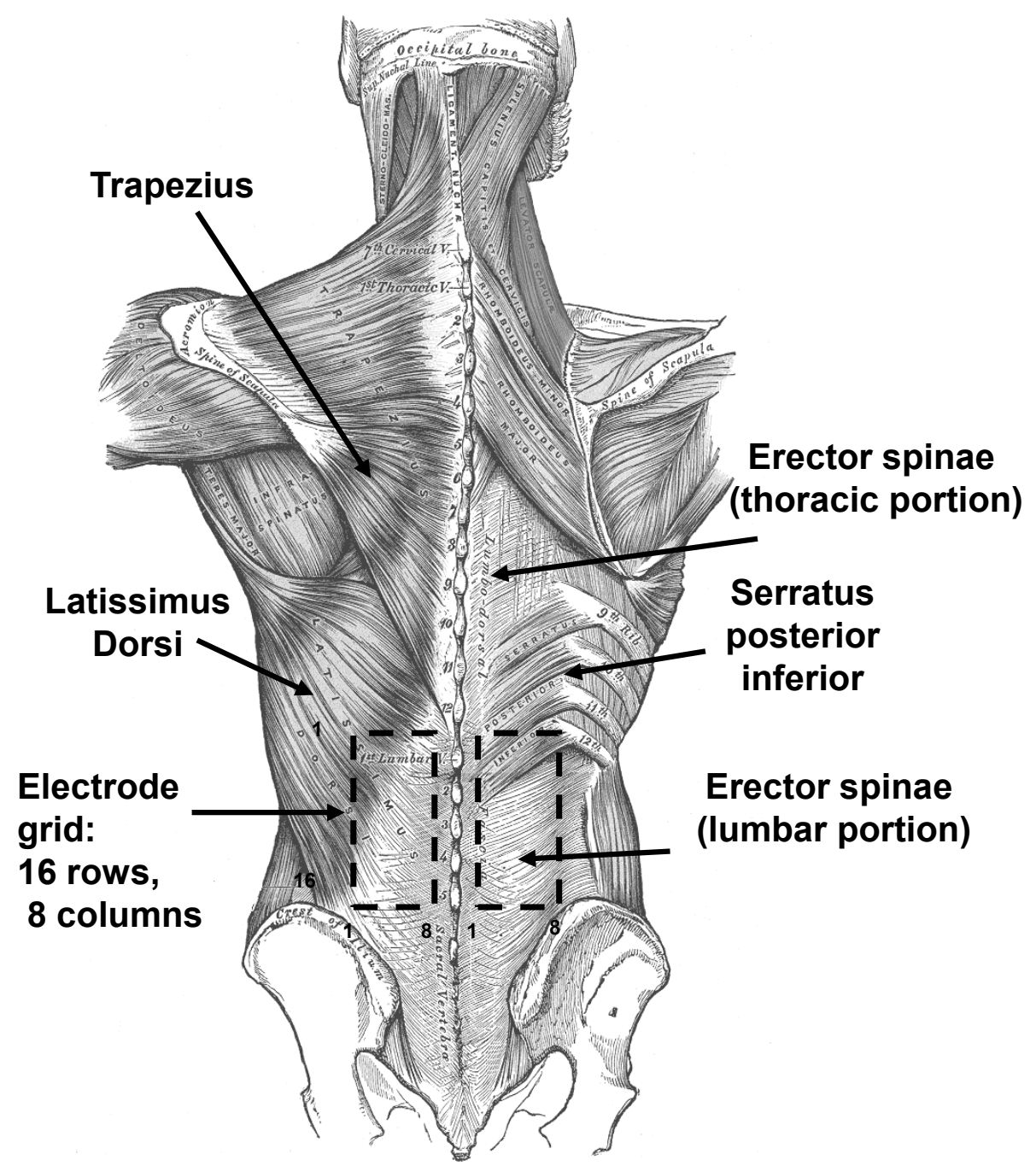

Fig 2 
a) Single differential signals from erector spinae, O-Chair, Column 7 (left side) and 2 (right side)

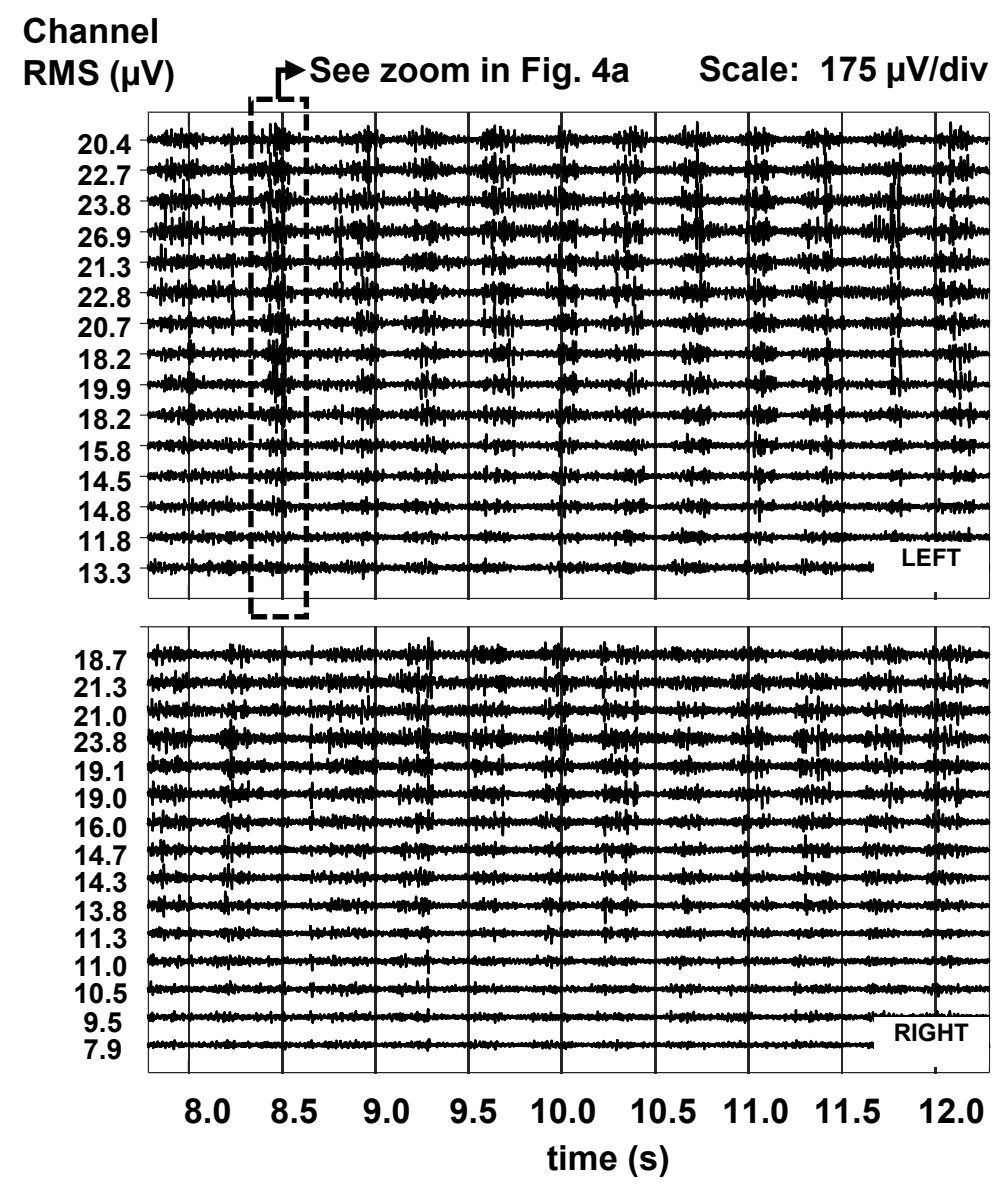

b) Single differential signals from erector spinae, A-Chair, Column 7 (left side) and 2 (right side)
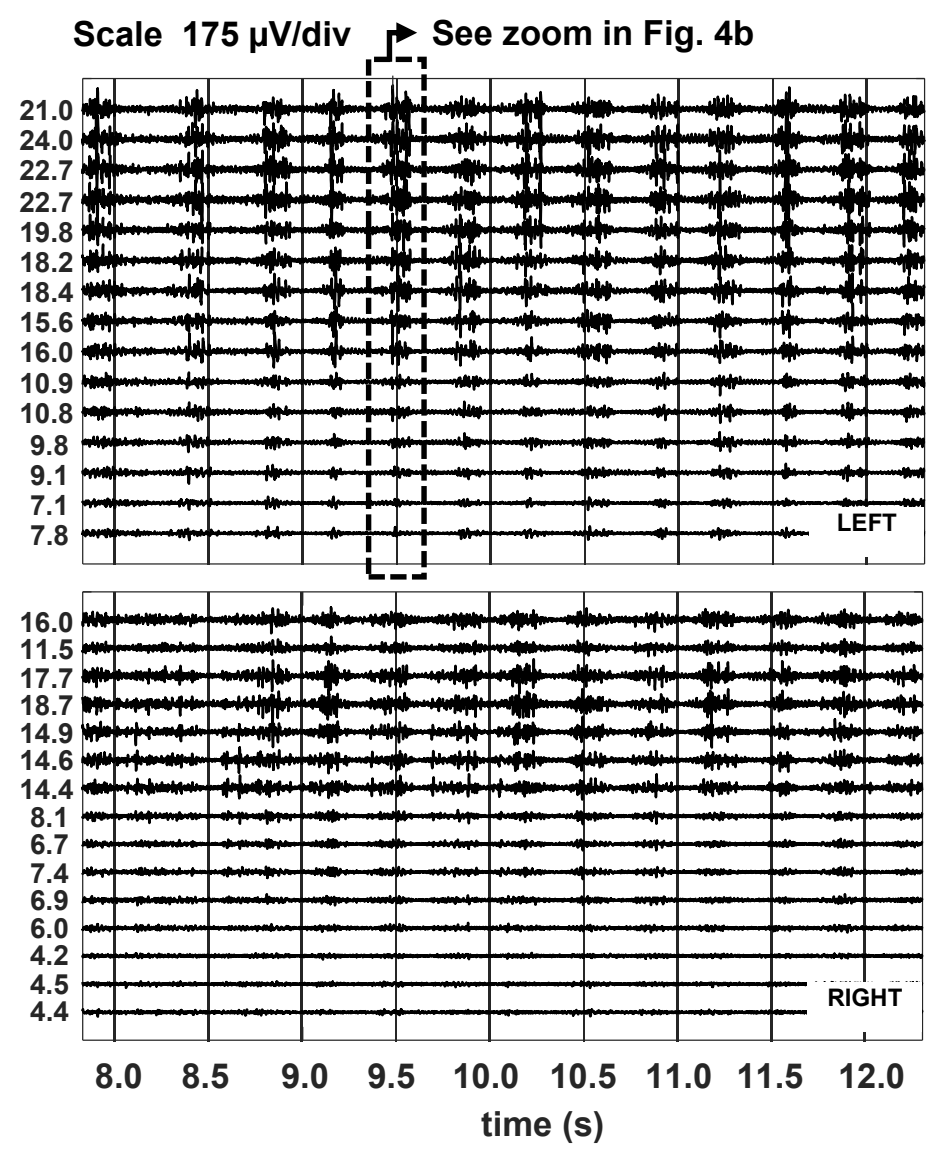

\section{Fig 3}


a) Single differential signals from erector spinae O-Chair, Zoom of a burst on column 7 (left side) b) Single differential signals from erector spinae, A-Chair, Zoom of a burst on column 7 (left side)
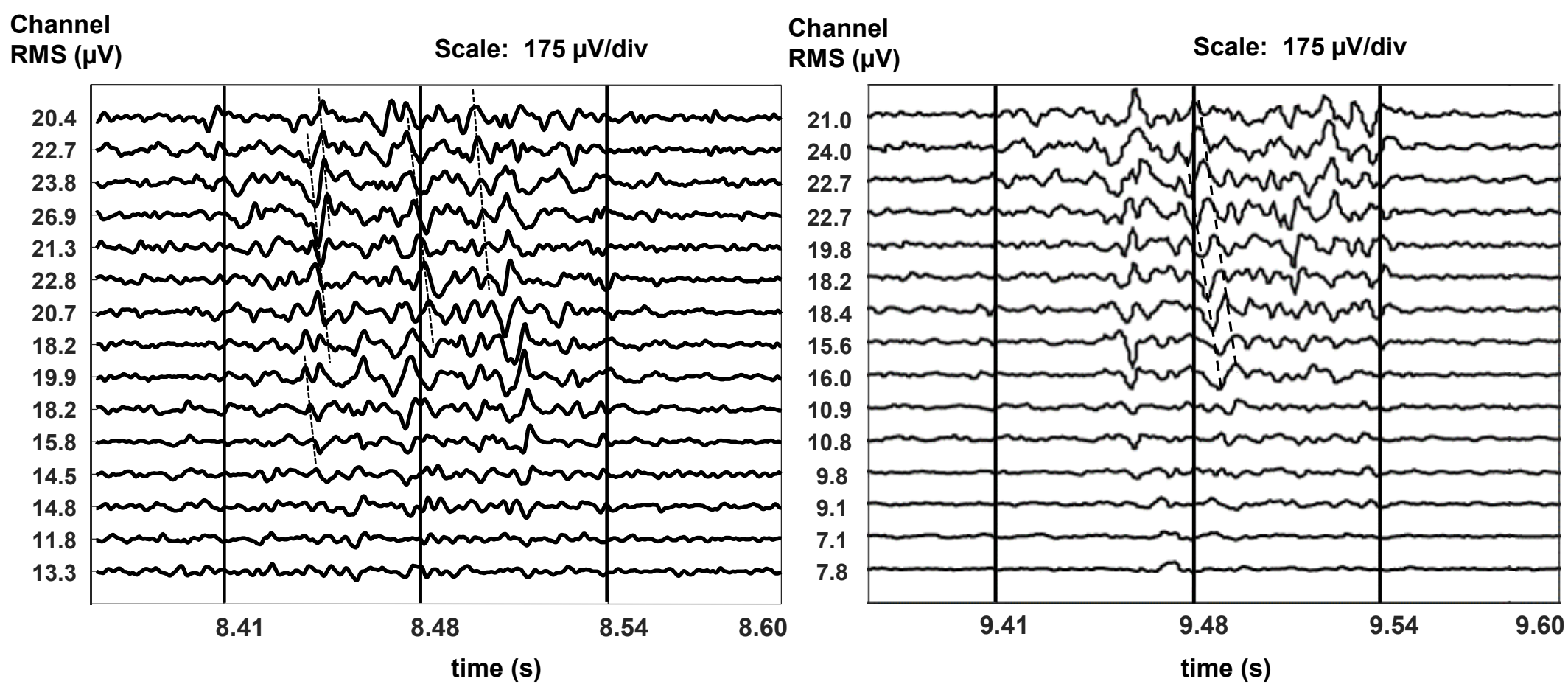

Fig 4 
Single differential RMS maps from a violin player calculated over a 20 s epoch

O-chair, 5 min

$19.3,13.0$ - 25.4

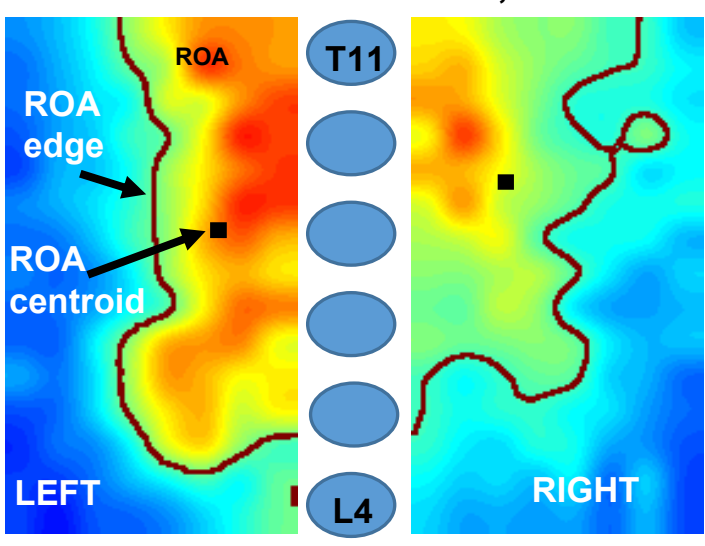

O-chair, $120 \mathrm{~min}$

$20.5,14.0-29.6$

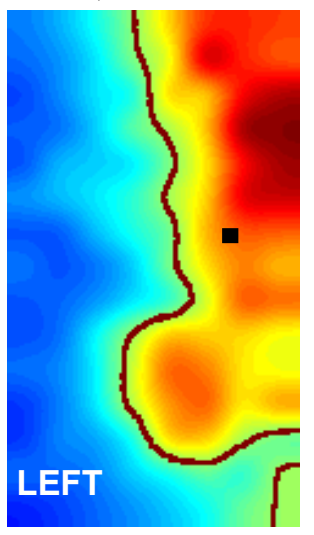

A-chair, 5 min

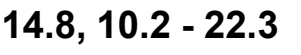

$12.9,8.7-19.4$
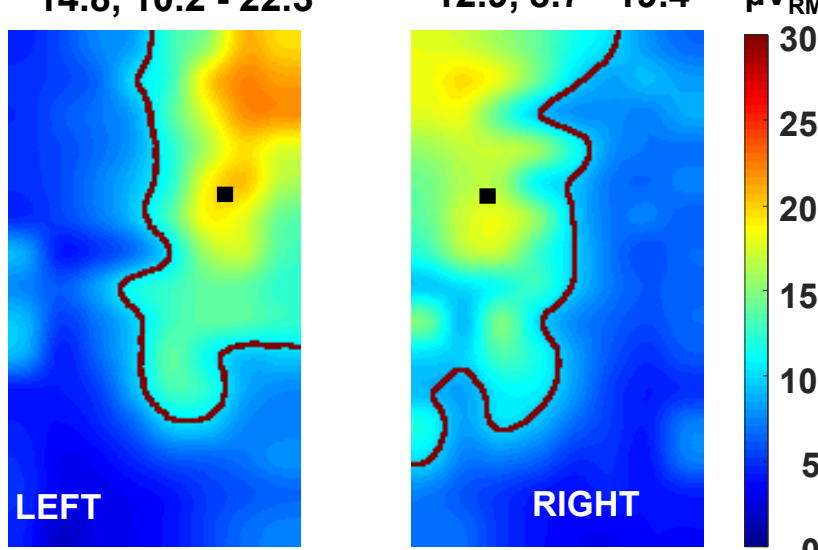

A-chair, 120 min

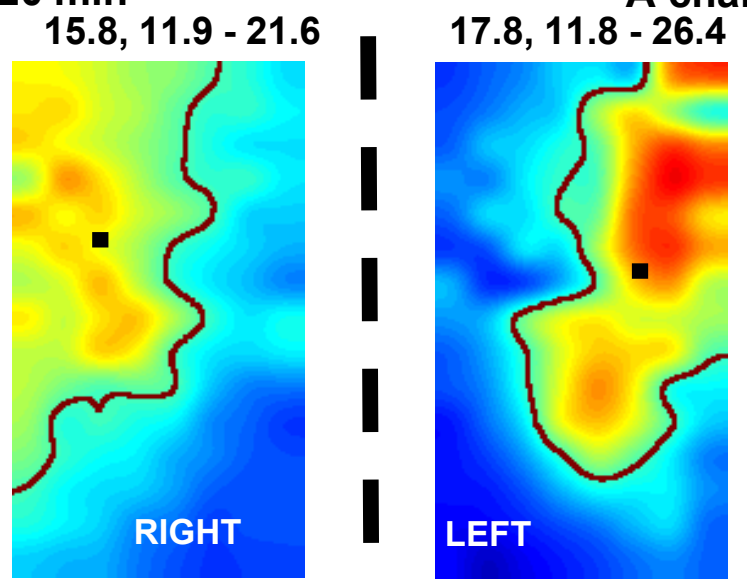

$10.4,4.2-19.3$

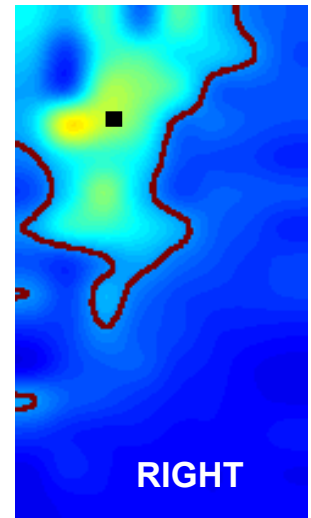

Fig. 5 


\section{Violin Players ( $N=9$ subjects)}

a) $\mathbf{O}$ - chair vs time

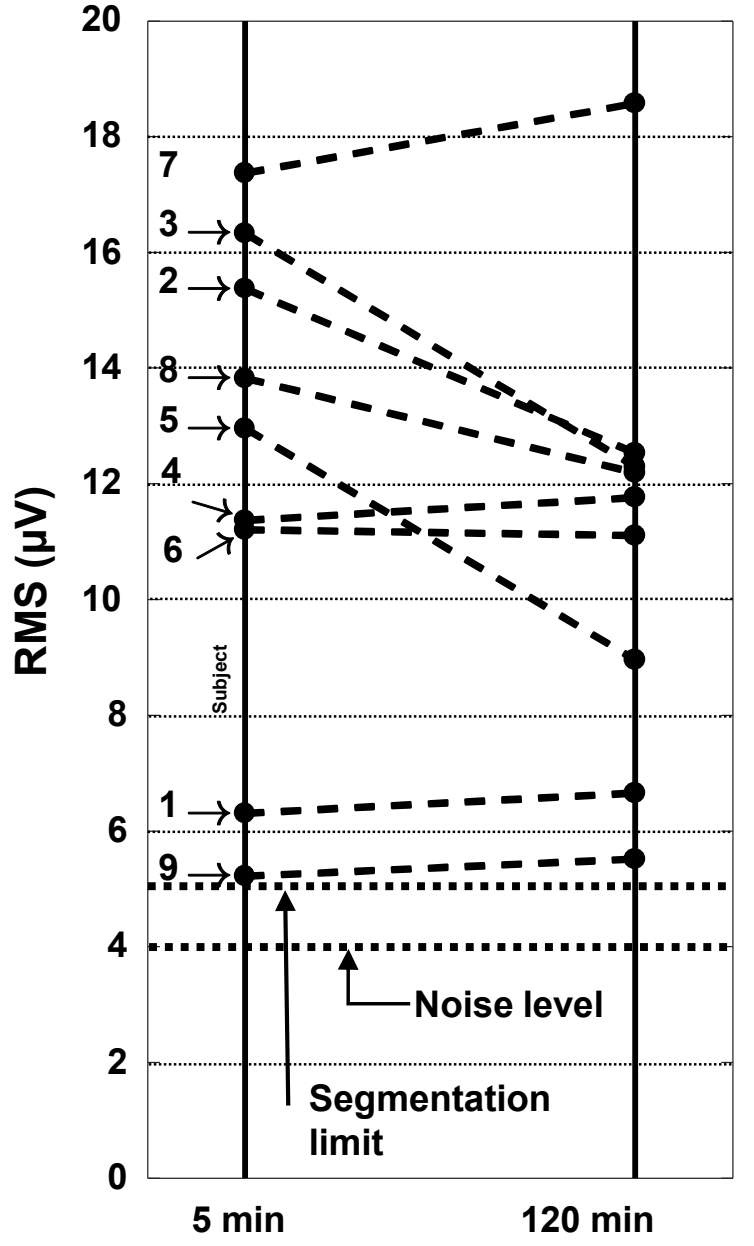

b) A - chair vs time

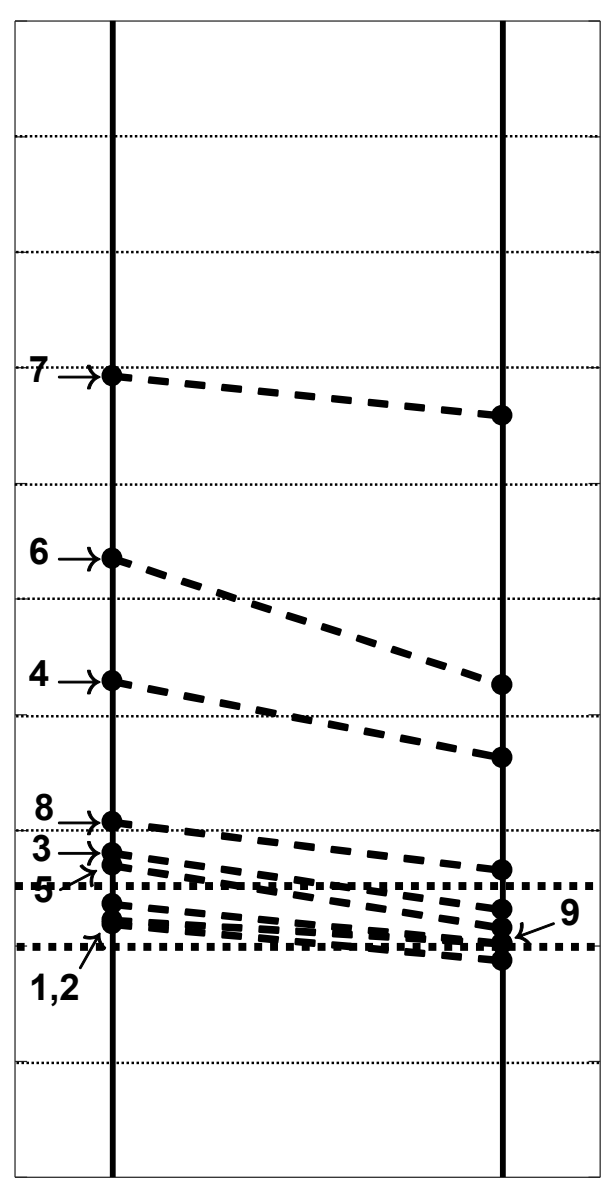

5 min c) $O$ chair vs $A$ chair

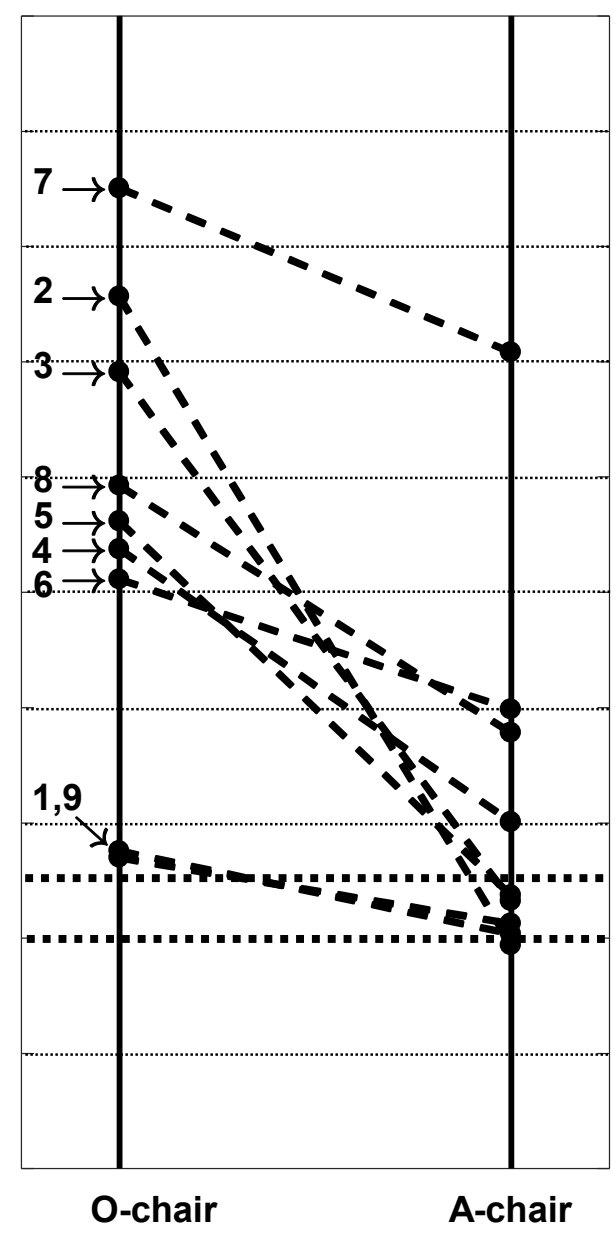

